function of generation time. Under many field conditions, species with long generation times experience more intense pesticidal selection per generation than species with short generation times. If toxins cause a constant mortality rate per day, then the time for resistance to appear is independent of generation time, and instead, selection intensity becomes a primary determinant of the rate of pest adaptation ${ }^{4}$. Detailed numerical simulations show that generation time can interact with ecological and genetic factors to produce a variety of influences on the rate of resistance evolution ${ }^{5}$.

Species with long generation times may in some cases experience such intense selection from insecticides that population densities decline catastrophically and local extinctions occur ${ }^{6}$. In this case, the evolution of resistance in long-lived species may be retarded beyond that expected from population genetics models in which infinite population sizes are assumed $^{7}$.

The extensive documentation of resistance evolution in arthropod pests provides an opportunity to test these different theoretical predictions. Our analysis of 586 North American arthropod pests of agriculture revealed no direct relationship between generation time and resistance

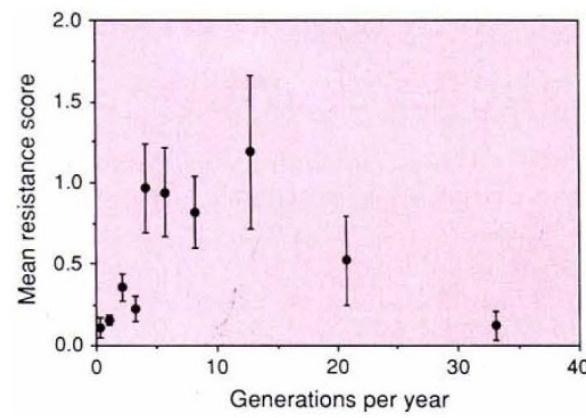

Resistance score (mean \pm s.e.; defined as the number of insecticide classes to which resistance has been documented in the field) for arthropod pests of agriculture in North America as a function of the number of generations per year completed by the pest. Data were analysed using a polychotomous stepwise logistic regression model; both the linear term (generations per year; $\chi^{2}=4.0, P$ $=0.04$; coefficient $=0.31 \pm 0.06$ ) and the quadratic term ([generations per year $]^{2} ; \chi^{2}=$ $20.5, P<0.0001$; coefficient $=-0.0098 \pm$ 0.0024 ) are significant. To facilitate data presentation, 10 intervals of generations per year are defined by the following cutoff points: $\leqslant 0.5(n=27) ; 1.5(243) ; 2.5$ (117); $3.5(83)$; 4.5 (29); 6.5 (33); 10.5 (22); 15.5 (16); 25.5 (21); and $>25.5$ (16). Values of generations per year were obtained from the literature. When estimates for the species in question were unavailable, average values for congeneric species were used ( $n=57$ ); for species in the families Aphididae and Tetranychidae, average values for confamilial species were used when congeners were unavailable $(n=20)$. evolution ${ }^{4}$. The tendency to evolve resistance to synthetic chemical insecticides as of 1980 was not linearly related to generation time; species with intermediate generation times (between four and ten generations per year) evolved resistance more readily than species with shorter or longer generation times ${ }^{4}$, but generation time explained only a very small fraction (5.7 per cent) of the total variation in resistance evolution.

Here we report that an enlarged and updated analysis of resistance evolution in North American arthropod pests confirms the result that resistance evolution does not depend directly upon generation time. The ability of each species $(n=607)$ to evolve resistance was quantified as the number of insecticide classes to which resistance had been reported up to 1989 (ref. 8). The simplest logistic regression model suggests that the number of generations per year makes no contribution to the ability to evolve resistance $\left(\chi^{2}=\right.$ 3.2, $P=0.08$ ). A more complete model that incorporates the influences of pest severity and pest feeding mode (chewing, sucking on phloem and xylem contents, or sucking on cell contents) identifies a significant curvilinear effect for generations per year; species with intermediate numbers of generations per year displayed the greatest ability to evolve resistance (see figure).

The relationship between the generation time of pests and their evolution of resistance to toxins in transgenic plants cannot be fully elucidated until such plants are widely deployed. Selection exerted by

toxins produced in transgenic plants may differ from selection exerted by conventional insecticides. Nonetheless, existing evidence from conventional insecticides suggests that the role of generation time may be limited, whereas factors directly linked to the intensity of selection, such as the concentration and temporal and spatial distribution of toxins, are likely to be critical. This result is important because it shifts the focus of attention from a biological trait that one generally cannot control (generation time) to parameters that will be shaped directly by the manner in which insecticidal transgenic plants are deployed.

Jay A. Rosenheim

Department of Entomology,

University of California,

Davis, California 95616, USA

Bruce E. Tabashnik

Department of Entomology,

University of Hawaii,

Honolulu,

Hawaii 96822, USA

1. May, R. M. Nature 361, 593-594 (1993).

2. May, R. M. \& Dobson, A. P. in Pesticide Resistance Strategies and Tactics for Management 170-193 (National Academy. Washington. DC, 1986)

3. Comins, H. N. in Genetics in Relation to Insect Management (eds Hoy, M. A. \& McKelvey, J. J. Jr) 55-69 (Rockefeller Foundation, New York. 1979).

4. Rosenheim, J. A. \& Tabashnik, B. E. Am. Nat. 137 527-541 (1991).

5. Rosenheim, J. A. \& Tabashnik, B. E. J. econ. Ent. 83 1184-1193(1990).

6. Mallet, J. \& Luttrell, R. Southwestern Entomologist Suppl. 15. 201-212 (1991)

7. Caprio, M. A. \& Tabashnik, B. E. J. econ. Ent. 85. 611-620 (1992).

8. Georghiou, G. P. \& Lagunes-Tejeda, A. The Occurrence of Resistance to Pesticides in Arthropods (Food and Agriculture Organization, Rome, 1991).

\title{
Thermodynamics at boundaries
}

SIR - After showing that entropy is proportional to mass or volume for homogeneous materials, Maddox in News and Views ${ }^{1}$ addresses the question of how it is that, in a special case, the black hole, it turns out to be proportional to area rather than volume. The discrepancy appears to him quaint enough to welcome an explanation in which quantum mechanics, plus complex mathematical paraphernalia, are involved. But is the case of entropy proportional to area as uncommon as Maddox apparently believes, or something almost trivially common?

Take the case of the reversible association of two globular protein subunits, a subject that has occupied my attention for the past 10 years (ref. 2 and references cited therein). These associations are entropy driven, as uniformly found since Lauffer et al. ${ }^{3}$ discovered the first case in 1958. The only contribution to the entropy change on association comes from replacement of the bonds at the boundary between protein and water by bonds of protein with protein. Assuming equal numbers of bonds of a typical energy for each reacting surface, the total entropy change on association is proportional to the number of bonds and therefore to the area of subunit interaction.

From such considerations it is easy to see that the same will be true of all cases of isothermal chemical reaction of polyatomic molecules that involve the bonds of the atoms at the interface and leave others unchanged: energy and entropy changes will be proportional to the interactive area, not to the volume of the reactive entities. Thus thermodynamics at the boundary of a black hole seem in this respect no different, Clausius and Gibbs be blessed, than at any other boundary.

\section{Gregorio Weber}

Department of Biochemistry,

University of Illinois,

Urbana,

IIlinois 61801, USA

\footnotetext{
1. Maddox, J. Nature 365, 103 (1993).

2. Weber, G. J. phys. Chem. 97, 7108-7115 (1993)

3. Lauffer, M. A., Ansevin, A. T., Cartwright, A. T. \& Brinton, C. C. Nature 181, 1338-1339 (1958)
}

NATURE - VOL 365 - 28 OCTOBER 1993 